DOI: 10.12957/demetra.2018.33495

\title{
Residências multiprofissionais em saúde como fomentadoras da formação interprofissional: percepção de nutricionistas sobre as práticas colaborativas
}

\author{
Multiprofessional residences in health as promoters of interprofessional training: perception \\ of nutritionists about collaborative practices
}

Elizabeth Rose Nogueira de Albuquerque Maria da Conceição Carneiro Pessoa de Santana ${ }^{2}$

Rosana Aparecida Salvador Rossit ${ }^{3}$

1 Universidade Federal de Alagoas, Hospital Universitário Prof. Alberto Antunes. Maceió, AL, Brasil.

${ }^{2}$ Universidade Estadual de Ciências da Saúde de Alagoas, Curso de Fonoaudiologia. Maceió, AL, Brasil.

${ }^{3}$ Universidade Federal de São Paulo, Programa de Pós-graduação Interdisciplinar em Ciências da Saúde. Santos, SP, Brasil.

Este artigo é resultado do Trabalho de Conclusão de Residência do Programa de Residência Multiprofissional em Saúde da Família da Universidade de Ciências da Saúde de Alagoas.

Correspondência / Correspondence

Elizabeth Rose Nogueira de Albuquerque

E-mail: bel_albuquerque00@hotmail.com

\section{Resumo}

O papel formador do Sistema Único de Saúde nos processos de integração ensino-serviço, vem estabelecendo as Residências Multiprofissionais em Saúde (RMS) como uma de suas estratégias para o fortalecimento das políticas públicas de saúde, no Brasil, tendo como uma de suas principais interfaces a atuação interprofissional. O profissional nutricionista tem conquistado espaço nas equipes de saúde para contribuir com seu saber específico e, enquanto profissional da saúde, em todas as perspectivas da Rede de Atenção à Saúde. O estudo avaliou a percepção sobre a formação interprofissional e o desenvolvimento de competências para práticas colaborativas de nutricionistas residentes inseridos no contexto das Residências Multiprofissionais em Saúde do Nordeste Brasileiro. Tratase de um estudo exploratório, do tipo transversal com dados de uma realidade, de caráter descritivo-analítico e natureza quantitativa. Os dados foram coletados durante o ano de 2015, a partir de questionário eletrônico hospedado na ferramenta do Google Docs, através de escala atitudinal tipo Likert. O público alvo da pesquisa foi residentes do segundo ano das RMS dos nove estados do Nordeste. Para análise dos dados, foi utilizado o teste estatístico não-paramétrico de Mann-Whitney. Os resultados apontaram que os nutricionistas residentes apresentam a mesma compreensão que os demais residentes das outras categorias profissionais sobre o desenvolvimento das práticas colaborativas nas RMS. Observou-se que, mesmo diante dos desafios postos pelos programas de residências, os residentes consideram que as Residências Multiprofissionais em Saúde possibilitam o 
desenvolvimento de competências para uma prática colaborativa em saúde no contexto do SUS.

Palavras-chave: Relações Interprofissionais. Nutricionistas. Profissional da Saúde. Residência não médica não odontológica. Colaboração.

\section{Abstract}

The formative role of the Unified Health System in the processes of teaching-service integration has been establishing Multiprofessional Residences in Health (MRH) as one of its strategies for strengthening public health policies in Brazil, having the interprofessional performance as one of its main interfaces. Professional nutritionists have gained space in the health teams to contribute with their specific knowledge and, as a health professional, in all perspectives of the Health Care Network. The study evaluated the perception on interprofessional training and the development of competences for collaborative practices of resident nutritionists inserted in the context of the Multiprofessional Residencies in Health in the Brazilian Northeast. This is an exploratory cross-sectional study with data from a reality, descriptive-analytical character and quantitative nature. Data were collected during the year 2015 from electronic questionnaire hosted in the Google Docs tool, through Likert type attitudinal scale. The target audience of the survey was composed of residents of the second year of MRH in the Northeastern states. For statistical analysis, the non-parametric Mann-Whitney statistical test was used. The results showed that resident nutritionists have the same understanding as other residents of other professional categories about the development of collaborative practices in MRH. It was observed that, even in the face of the challenges posed by the residency programs, the residents consider that the Multiprofessional Residences in Health allow the development of competences for a collaborative practice in health in the SUS context.

Keywords: Interprofessional Relations. Nutritionists. Health Professional. Non-medical non-dental residency. Collaboration. 


\section{Introdução}

O Sistema Único de Saúde (SUS) tem papel formador nos processos de integração ensinoserviço, de acordo com seu arcabouço jurídico que, ao longo dos anos, vem estabelecendo uma série de políticas públicas voltadas para o fomento de programas e políticas inovadoras que viabilizem estratégias de formação e práticas profissionais para além do cuidado médico assistencial, que contemplem a efetivação das equipes de saúde responsáveis pelo cuidado, bem como estimulando a corresponsabilização do usuário com sua saúde. Nesta perspectiva, o redirecionamento da gestão de profissionais no âmbito do SUS e em todo o escopo abrangido pela lei 8.080 e demais legislações, propõem uma mudança na formação inicial, de pós-graduação e no contexto dos serviços através da Educação Permanente em Saúde e das práticas do trabalho em equipe. ${ }^{1-4}$

A Organização Mundial da Saúde (OMS), a partir da publicação do Marco para Ação em Educação Interprofissional e Prática Colaborativa, aponta que a qualificação dos serviços de saúde ofertados aos usuários é potencializada pela perspectiva interprofissional no cotidiano dos serviços e também na graduação e pós-graduação dos trabalhadores da saúde. Logo, o fortalecimento do trabalho interprofissional favorece a implementação da prática colaborativa em saúde, que ao fomentar a comunicação para a tomada de decisões, estabelece a consolidação do cuidado integral. ${ }^{4,5}$

Desta forma, a formação interprofissional em saúde tem se caracterizado um desafio a ser superado pelas novas gerações com a finalidade de adequar as demandas dos serviços às necessidades da atenção integral à saúde. Por ser uma proposta inovadora e em constate transformação, o conceito de interprofissionalismo não possui um consenso entre os autores, porém perpassa uma concepção e estratégia onde duas ou mais profissões são orientadas pelo trabalho em equipe onde exista uma prática colaborativa e de aprendizagem conjunta sobre as especificidades de cada um e entre si, decorrendo o respeito e sem qualquer tipo de hierarquia entre os profissionais durante sua formação e no contexto dos serviços. ${ }^{2,5,6}$

Diversos autores têm se dedicado a evidenciar que a educação interprofissional desenvolvida durante a graduação por meio da interdisciplinaridade e das práticas supervisionadas em serviço tem traçado movimentos positivos onde estes estudantes/profissionais em formação, não abrindo mão de sua formação específica, possuam capacidade crítica e reflexiva para lidar com essa proposta educacional relativamente recente. Desta forma, percebe-se que o interprofissionalismo deve iniciar na graduação, cujo papel é de preparar, com currículos cada vez mais inovadores, profissionais capazes de atuar de forma integrada e em equipe, além de lidar com as demandas dos serviços de saúde, considerando o SUS como ordenador da formação. ${ }^{2,7,8}$

Impulsionadas durante o contexto da Reforma Sanitária, as primeiras experiências de Residências Multiprofissionais em Saúde (RMS) surgiram a partir de 1999, regulamentadas alguns anos depois a partir de sua exitosa contribuição para os serviços de saúde, conquistando sua 
promulgação em 2005 através da lei n ${ }^{\circ} 11.129$. As RMS foram pensadas dentro de uma perspectiva de cooperação intersetorial entre Ministério da Saúde e Ministério da Educação, desenvolvidas considerando as áreas prioritárias do SUS. São um modelo de formação direcionados atualmente com os princípios da formação interprofissional: interdisciplinaridade, cuidado integral e clínica ampliada, a partir da uma proposta de aprendizagem conjunta e de práticas colaborativas impulsionadas pelo cotidiano dos serviços, na busca pelo fortalecimento do processo de trabalho com foco no usuário. ${ }^{4,9-13}$

O atual perfil epidemiológico da população brasileira, a partir das condições crônicas não transmissíveis, requer um acompanhamento a longo prazo e com uma dimensão de cuidado que só pode ser contemplado com uma abordagem integral. Desta forma, a interprofissionalidade vem responder a tais demandas e fomentar a discussão da prática colaborativa em saúde, sendo a integração de vários núcleos profissionais norteadoras desse processo de cuidado. Nesta mesma perspectiva, considerando o perfil epidemiológico brasileiro, o profissional nutricionista tem composto cada vez mais as equipes multiprofissionais de saúde para contribuir com seu saber específico e enquanto profissional da saúde em todas as perspectivas da Rede de Atenção à Saúde. ${ }^{4,6,14}$

Desta forma, os cursos de graduação em nutrição têm sentido a necessidade de (re)adequar suas diretrizes curriculares ao contexto do trabalho em equipe e das práticas colaborativas para fomentar este tipo de atuação pelos nutricionistas, tendo iniciado estes esforços a partir de 2001 através das Diretrizes Curriculares Nacionais (DCNs). Estas são normas para todos os níveis da educação brasileira que orientam o planejamento curricular e são obrigatórias nos cursos de graduação. Por meio do Conselho Nacional de Educação (CNE) elas são elaboradas, discutidas e fixadas para promover equidade da aprendizagem e possibilitar a uniformização dos cursos pelas Instituições de Ensino Superior (IES). Em 2001, houve a instituição das Diretrizes Curriculares Nacionais do Curso de Nutrição através da resolução CNE/CES no 5, de 7 de novembro. Estas vieram definir os princípios, fundamentos, condições e procedimentos da formação de nutricionistas com formação generalista, humanista e crítica, pautado em princípios éticos, com reflexão sobre a realidade econômica, política, social e cultural. . $^{15,16}$

A Política Nacional de Alimentação e Nutrição (PNAN) tem proposto, neste mesmo direcionamento, uma atenção nutricional coparticipativa dos profissionais de saúde, e não apenas dos nutricionistas, incluindo uma atuação intersetorial a partir dos desafios que são enfrentados no campo da alimentação e nutrição no SUS. Esta Política vem desde 1999 articulando e propondo ações para o cumprimento do direito constitucional da alimentação e o fortalecimento da atenção nutricional, a partir da Atenção Básica, com foco na vigilância, promoção, prevenção e cuidado integral de agravos relacionados à alimentação e nutrição. ${ }^{14,17,18}$ 
Assim, este estudo teve por objetivo avaliar a percepção sobre a formação interprofissional e o desenvolvimento de competências para práticas colaborativas de nutricionistas residentes inseridos no contexto das Residências Multiprofissionais em Saúde do Nordeste Brasileiro.

\section{Materiais e Métodos}

Trata-se de um estudo exploratório, do tipo transversal com dados de uma realidade, de caráter descritivo-analítico e natureza quantitativa. Ressalta-se que esta pesquisa foi um recorte de uma tese de doutorado em andamento sobre o Interprofissionalismo nas Residências Multiprofissionais em Saúde do Nordeste Brasileiro. Assim, a finalidade foi avaliar a formação interprofissional e o desenvolvimento de competências para práticas colaborativas apenas dos residentes, comparando os profissionais nutricionistas às demais categorias profissionais inseridas neste contexto (assistentes sociais, cirurgiões-dentistas, enfermeiros, farmacêuticos, fisioterapeutas, fonoaudiólogos, médicos veterinários, profissionais de educação física, psicólogos e terapeutas ocupacionais).

Os dados foram coletados durante o ano de 2015 através de questionário eletrônico hospedado na ferramenta do GoogleDocs e compartilhado via redes sociais e endereço eletrônico. O instrumento de coleta de dados para avaliar a formação interprofissional foi estruturado e validado por Perego ${ }^{12}$ em 2015. O questionário de 21 asserções apresentou confiabilidade de 93\% e uma escala atitudinal tipo Likert, avaliando as seguintes dimensões: Aprendizagens Compartilhadas na Residência Multiprofissional, Formação para o Trabalho em Equipe e Desenvolvimento de competências para Práticas Colaborativas. Para este estudo foi considerada a última dimensão sendo essa relacionada a 7 asserções.

O público alvo da pesquisa principal foram os residentes do segundo ano e docentes (coordenadores, tutores e preceptores) das Residências Multiprofissionais em Saúde do Nordeste Brasileiro, sendo consideradas multiprofissionais, os programas de residências (de quaisquer modalidades: saúde da família, zona rural, hospitalar, entre outras) que apresentassem no mínimo três categorias profissionais, conforme a Resolução do Conselho Nacional de RMS. ${ }^{19}$ Para este estudo foram considerados apenas os profissionais residentes (nutricionistas e demais residentes). Observada a Resolução 466/2012, ${ }^{20}$ do Conselho Nacional de Saúde, a pesquisa foi aprovada pelo Comitê de Ética e Pesquisa da Universidade Federal de São Paulo (Unifesp), sob parecer no 1.094.027, de 03/06/2015, Certificado de Apresentação para Apreciação Ética (CAAE): 45075815.7.0000.5505, onde todos os participantes assinalaram consentimento através do Termo de Consentimento Livre e Esclarecido (TCLE), sendo este em formato on-line, compondo o instrumento utilizado, onde o participante da pesquisa somente poderia responder ao questionário após estar ciente do descrito no TCLE. Para delimitação do público, foi considerado o Sistema Nacional de Residências Multiprofissionais do Ministério da Educação que apresenta todas as Residências cadastradas por estado brasileiro. Foram consideradas todas as RMS dos nove Estados do Nordeste. 
Do público de 366 respondentes à pesquisa, 64,75\% (n237) deles eram residentes e destes, 10,13\% (n24) possuem graduação em Nutrição, enquanto que 89,87\% (n213) possuem graduação nas demais categorias profissionais já apresentadas. Foram incluídos neste recorte todos os residentes (nutricionistas e outros residentes). Para análise dos dados foram utilizados os recursos dos softwaresExcel2010® e Bioestat5.0® com aplicação de teste estatístico não-paramétrico de Mann-Whitney e quanto à significância estatística, foi considerado o valor de $\mathrm{p}<0,05$. Para a Escala do tipo Likert foram considerados as seguintes pontuações para cada escala: Concordo Totalmente (4 pontos); Concordo (3 pontos); Discordo (2 pontos); Discordo Totalmente (1 ponto).

\section{Resultados e Discussão}

O instrumento utilizado abordou além das asserções, através de escala atitudinal, o perfil dos participantes considerando as seguintes questões apresentadas nas tabelas 1 e 2 . Foi considerado 'NR' para nutricionistas residentes e 'OR' para os outros residentes das demais categorias profissionais. Foi considerado também as seguintes siglas para cada escala: Concordo Totalmente (CT), Concordo (C), Discordo (D) e Discordo Totalmente (DT).

Tabela 1. Caracterização dos sujeitos da pesquisa por gênero e idade, 2017.

\begin{tabular}{|c|c|c|c|c|}
\hline & NR & OR & NR & OR \\
\hline Gênero & $\mathrm{n}$ & $\mathrm{n}$ & $\%$ & $\%$ \\
\hline Masculino & 01 & 38 & $4,17 \%$ & $17,84 \%$ \\
\hline Feminino & 23 & 175 & $95,83 \%$ & $82,16 \%$ \\
\hline \multicolumn{5}{|l|}{ Idade } \\
\hline $20 \vdash 25$ & 04 & 33 & $16,67 \%$ & $15,49 \%$ \\
\hline $25 \vdash 30$ & 17 & 162 & $70,83 \%$ & $76,06 \%$ \\
\hline $30 \vdash 35$ & 02 & 10 & $8,33 \%$ & $4,69 \%$ \\
\hline $35 \vdash 40$ & 00 & 06 & $0 \%$ & $2,82 \%$ \\
\hline $40 \vdash 50$ & 01 & 02 & $4,17 \%$ & $0,94 \%$ \\
\hline
\end{tabular}


Tabela 2. Caracterização dos sujeitos da pesquisa por estado, ano de formação e formação em instituição pública ou privada, 2017.

\begin{tabular}{|c|c|c|c|c|}
\hline & NR & OR & NR & OR \\
\hline Estado & $\mathbf{n}$ & $\mathbf{n}$ & $\%$ & $\%$ \\
\hline Alagoas & 3 & 29 & $12,5 \%$ & $13,62 \%$ \\
\hline Bahia & 6 & 20 & $25 \%$ & $9,39 \%$ \\
\hline Ceará & 4 & 51 & $16,67 \%$ & $23,94 \%$ \\
\hline Maranhão & 4 & 51 & $16,67 \%$ & $23,94 \%$ \\
\hline Paraíba & 1 & 7 & $4,17 \%$ & $3,29 \%$ \\
\hline Pernambuco & 1 & 25 & $4,17 \%$ & $11,74 \%$ \\
\hline Piauí & 3 & 14 & $12,5 \%$ & $6,57 \%$ \\
\hline Rio Grande do Norte & 2 & 6 & $8,33 \%$ & $2,82 \%$ \\
\hline Sergipe & 0 & 10 & - & $4,69 \%$ \\
\hline \multicolumn{5}{|l|}{ Ano de Formação } \\
\hline $2001 \vdash 2009$ & 1 & 7 & $4,17 \%$ & $3,29 \%$ \\
\hline $2009 \vdash 2011$ & 2 & 17 & $8,33 \%$ & $7,98 \%$ \\
\hline $2011 \vdash 2013$ & 7 & 59 & $29,17 \%$ & $27,7 \%$ \\
\hline $2013 \vdash 2015$ & 14 & 127 & $58,33 \%$ & $59,62 \%$ \\
\hline Erro na Informação & 0 & 3 & - & $1,41 \%$ \\
\hline \multicolumn{5}{|l|}{ Instituição Formadora } \\
\hline Egresso Pública & 19 & 148 & $79,17 \%$ & $69,48 \%$ \\
\hline Egresso Privada & 5 & 64 & $20,83 \%$ & $30,05 \%$ \\
\hline Erro na Informação & 0 & 1 & - & $0,47 \%$ \\
\hline
\end{tabular}

Quanto ao perfil dos residentes nutricionistas, estes apresentaram média de idade de 26 anos, sendo apenas um dos residentes do sexo masculino. O estado do Nordeste que apresentou o maior número de respondentes foi a Bahia (25\%), enquanto que Sergipe foi o único estado que não participou da pesquisa nesta categoria profissional. Sobre o ano de conclusão da graduação, $75 \%$ dos nutricionistas residentes finalizaram sua formação acadêmica nos últimos cinco anos e a maior parte dos nutricionistas residentes são egressos de universidades públicas $(79,17 \%)$. 
Entre as outras categorias profissionais, a média de idade apresentada também foi de 26 anos, sendo 82,16\% destes do sexo feminino. O estado que apresentou maior número de respondentes foi o Ceará e o Maranhão com 23,94\% cada, enquanto que o estado de Sergipe novamente teve baixa representação (4,69\%). A respeito do ano de conclusão da graduação, 73,24\% destes concluíram nos últimos cinco anos e 69,48\% dos residentes de outras categorias profissionais, são egressos de universidades públicas.

O quantitativo de respostas por asserções com escala tipo Likert e os resultados do p-valor para análise do nível de significância são apresentados nas tabelas 3 e 4.

Tabela 3. Comparações das respostas das asserções: (A1), (A3), (A7), (A9), (A10), (A14), (A19) entre os residentes nutricionistas e demais categorias de residentes do nordeste do Brasil, 2017.

\begin{tabular}{lcccc}
\hline Asserção & & Mediana & DIQ & p-valor \\
\hline A1 & Nutricionistas & 4 & 1 & 0,5708 \\
\hline Outros & Nutricionistas & 3 & 1 & \\
\hline Outros & O,5 & 3 & 1 & 0,9862 \\
\hline Nutricionistas & Outros & 4 & 0 & 0,7168 \\
\hline A9 & Nutricionistas & 3 & 1 & 0,763 \\
& Outros & 4 & 1 & \\
\hline A10 & Nutricionistas & 4 & 1 & 0,3334 \\
\hline O19 & Outros & 4 & 1 & 0,5382 \\
\hline & Nutricionistas & 4 & 1 & \\
\hline & Outros & 3,5 & 1 & 1 \\
\hline
\end{tabular}


Tabela 4. Escala atitudinal com as respostas das asserções 1, 3, 7, 9, 10, 14 e 19, referentes à dimensão das práticas colaborativas em saúde dos nutricionistas residentes e outros residentes, 2017.

\begin{tabular}{ccccccccc}
\hline Asserção & \multicolumn{2}{c}{ CT } & & C & \multicolumn{2}{c}{ D } & \multicolumn{2}{c}{ DT } \\
\hline NR & OR & NR & OR & NR & OR & NR & OR \\
\hline A3 & $58 \%$ & $50 \%$ & $38 \%$ & $49 \%$ & $4 \%$ & $1 \%$ & $0 \%$ & $0 \%$ \\
A7 & $50 \%$ & $49 \%$ & $46 \%$ & $49 \%$ & $4 \%$ & $2 \%$ & $0 \%$ & $0 \%$ \\
A9 & $62 \%$ & $49 \%$ & $42 \%$ & $48 \%$ & $4 \%$ & $2 \%$ & $0 \%$ & $1 \%$ \\
A10 & $67 \%$ & $56 \%$ & $33 \%$ & $40 \%$ & $0 \%$ & $3 \%$ & $0 \%$ & $1 \%$ \\
A14 & $50 \%$ & $43 \%$ & $42 \%$ & $50 \%$ & $8 \%$ & $7 \%$ & $0 \%$ & $0 \%$ \\
A19 & $67 \%$ & $60 \%$ & $33 \%$ & $38 \%$ & $0 \%$ & $2 \%$ & $0 \%$ & $0 \%$ \\
\hline
\end{tabular}

Os resultados de p-valor, com aplicação do teste de estatístico de Mann-Whitney, concluem que não há diferença significativa entre as respostas dos residentes nutricionistas com as respostas dos outros residentes em todas as asserções. Significa dizer que os nutricionistas residentes apresentam a mesma compreensão sobre o desenvolvimento das práticas colaborativas nas RMS que os demais residentes das outras categorias profissionais.

A asserção 1 (Al) questionou se a residência multiprofissional possibilita o desenvolvimento de competências para uma prática colaborativa entre os residentes, potencializando suas habilidades e pontos fortes com uma visão ampliada de saúde, tendo como concordância total de $58 \%$ dos nutricionistas residentes (NR) e de $50 \%$ dos outros residentes (OR). A asserção 3 (A3) examinou a concepção dos residentes diante da afirmativa das práticas colaborativas não se referirem somente a acordo e comunicação, mas à criação de sinergia, pois ocorre quando há interação entre as diferentes profissões para criar uma compreensão compartilhada. A concordância total da A3 foi de $50 \%$ dos NR e $49 \%$ dos OR.

Diante do questionamento de que a integração dos profissionais de saúde na residência multiprofissional possibilita uma postura de compartilhamento na prática em serviço, a asserção 7 (A7) apresentou concordância total de $54 \%$ dos NR e de 49\% dos OR. Já a análise da questão de que a prática colaborativa no trabalho em equipe possibilita uma atuação integrada entre os profissionais de saúde, produzindo melhores resultados em saúde, exibiu o resultado de concordância total da maioria dos residentes, sendo $62 \%$ dos NR e de $60 \%$ dos OR para a assertiva 9 (A9). 
A assertiva 10 (A10) analisou se para uma prática colaborativa eficaz na residência multiprofissional são necessários mecanismos como uma força de trabalho colaborativa, apoios institucionais e cultura de trabalho e ambiente, tendo como resultado de concordância total de $67 \%$ dos NR e 56\% dos OR. A avaliação de que a residência multiprofissional prepara os residentes para um processo de trabalho colaborativo, ampliando a visão do processo saúde e doença, apresentou concordância total de $50 \%$ dos NR e a menor concordância total dos OR, 43\%. E, finalmente, na assertiva 19 (A19), que questionou se maior grau de satisfação dos pacientes, melhor aceitação da assistência e melhores resultados de saúde são encontrados após o cuidado prestado por uma equipe de saúde colaborativa, apresentou o melhor resultado de concordância total dos residentes: $67 \%$ dos nutricionistas residentes e $60 \%$ dos outros residentes.

\section{O desenvolvimento de competências para Práticas Colaborativas}

A prática colaborativa entre os residentes foi apontada como potencializadora de suas habilidades e pontos fortes com uma visão ampliada de saúde. Silva ${ }^{3}$ aponta que a visão ampliada de saúde possibilita a proposta da clínica ampliada como uma prática colaborativa. Assim, Araújo ${ }^{4}$ em 2017, corrobora evidenciando que não apenas os residentes, como os docentes também apontam o contexto da clínica ampliada como uma das grandes aprendizagens da conjuntura da RMS, a qual fomenta as práticas de colaboração entre os profissionais através do cenário prático e teórico entre os residentes e entre os docentes e residentes.

Característica marcante das entrevistas realizadas por Oliveira ${ }^{21}$ com estudantes de graduação da área saúde, incluindo estudantes de nutrição, que tem tido uma formação acadêmica com proposta interprofissional, foi que esse tipo de formação proporciona a prática do diálogo e da escuta com respeito às diferenças, confirmando o preparo para o trabalho em equipe. Da mesma forma, na presente pesquisa ambos os grupos analisados concordaram que as práticas colaborativas não se referem somente a acordo e comunicação, mas à criação de sinergia, pois ocorre quando há interação entre as diferentes profissões para criar uma compreensão compartilhada.

Outro aspecto considerado foi de que a integração dos profissionais de saúde na Residência Multiprofissional possibilita uma postura de compartilhamento na prática em serviço. Da mesma forma, Rodrigues ${ }^{22}$ em 2016, discute que essa integração possibilita a construção concreta e efetiva do trabalho com os profissionais do serviço, movimentando uma rede composta por todos os trabalhadores e não apenas pelos profissionais inseridos no contexto das RMS.

Os residentes (nutricionistas e de outras categorias) chegaram a mesma conclusão ao considerarem a prática colaborativa no trabalho em equipe fomentadora de uma atuação integrada entre os profissionais de saúde, produzindo melhores resultados em saúde. Costa ${ }^{23}$ e Reeves $^{24}$ apresentaram discussões que vem sendo debatidas nas últimas décadas em relação à Educação Interprofissional, 
comprovando que esta tem impactado sistemas de saúde em todo o mundo, apontando para uma articulação intencional e resultando em ações mais resolutivas da atenção à saúde.

Ambos grupos da pesquisa (nutricionistas residentes e outros residentes) apresentaram os mesmos resultados ao concordarem que para uma prática colaborativa eficaz na Residência Multiprofissional, são necessários mecanismos como uma força de trabalho colaborativa, apoios institucionais e cultura de trabalho e ambiente. O profissional nutricionista tem sido incorporado gradativamente nas RMS e nos demais espaços de fortalecimento do trabalho em equipe no SUS desde os primeiros esforços destes modelos de trabalho, porém, ainda com número insuficiente de profissionais. ${ }^{18}$ Recine $^{25}$ em 2014 elenca uma série de desafios no contexto do trabalho do nutricionista e entre eles destaca-se os "saberes e práticas em suas relações com a saúde coletiva e as demais ciências" e principalmente a "tradução do conhecimento em prática ampla e efetiva, principalmente na atenção básica do SUS".

O cotidiano dos serviços tem necessitado de implicações práticas sobre o processo saúde e doença, para além das discussões teóricas na academia, ampliando a visão dos profissionais de saúde para este complexo processo. Segundo os dados apresentados nesta pesquisa, os residentes apontaram que a Residência Multiprofissional tem organizado estes para um processo de trabalho colaborativo, ampliando a visão do processo saúde e doença. ${ }^{26}$ Tal resultado é reafirmado por outro estudo que considera as RMS como um dispositivo que oportuniza os profissionais de saúde, ainda em formação, a participar e provocar um trabalho colaborativo, de educação permanente em saúde e singular, com olhar integrado e ampliado para o processo saúde e doença. ${ }^{27}$

A pesquisa também constatou que a análise do grau de satisfação dos pacientes é maior quando o cuidado é prestado por uma equipe de saúde colaborativa, uma vez que propicia melhor aceitação da assistência e melhores resultados de saúde. Estudo realizado em 2013, ao analisar a expectativa e a satisfação dos usuários referente aos serviços oferecidos pelas equipes da Estratégia Saúde da Família de um município do sul de Goiás, considerou a importância do olhar do usuário frente ao cuidado que é prestado pelas equipes de saúde, uma vez que sua satisfação com o cuidado que lhe é prestado configura-se como um importante fator de adesão do usuário à terapêutica e à corresponsabilização de sua saúde. ${ }^{28}$

\section{O Nutricionista no contexto das RMS e as Práticas Colaborativas}

O Nutricionista é o profissional da saúde responsável pelo cuidado e todas as interfaces concernentes à alimentação e nutrição do indivíduo e populações. A profissão foi criada através de lei em 1967 e regulamentada em 1991 pela lei 8.234/199129 e desde então vem sendo reconhecida pela sociedade em suas diversas áreas de atuação, inclusive nas equipes de saúde do SUS. As RMS, ao visar a qualificação dos profissionais de saúde para o SUS, possibilita a inserção dos nutricionistas 
neste contexto e amplia as potencialidades da formação de um profissional da saúde para além das suas competências profissionais exclusivas, mas também para questões e diálogos interprofissionais através das aprendizagens compartilhadas, trabalho em equipe e das práticas colaborativas. ${ }^{12,30,31}$

Souza $^{30}$ ao analisar a inserção do nutricionista em residências multiprofissionais, observou que o trabalho interdisciplinar nesta conjuntura, propicia uma atuação prática e de novos saberes, oportunizado pelo convívio colaborativo. $\operatorname{Santos}^{31}$, ao investigar a concepção dos profissionais de saúde em relação à atuação do nutricionista na Estratégia Saúde da Família após sua inserção em um programa de RMS, concluiu que os profissionais percebem o nutricionista como um agente de promoção à saúde e prevenção de agravos, além de valorizarem seu conhecimento específico. Concluiu também que após a passagem por uma RMS, ao nutricionista era possibilitado uma visão a atuação ampliada de saúde com vistas ao interprofissionalismo.

\section{Considerações finais}

O sistema público de saúde no Brasil vem passando por um processo de desmonte e ameaça de suas políticas, onde o SUS, construído através de resistência e capacidade de atuação das forças sociais e políticas, enfrenta desafios importantes, atualmente e para os próximos anos. Assim, fortalecer a força de trabalho do sistema parece necessário para o enfrentamento desse cenário, sendo uma das grandes apostas das políticas públicas de saúde a atuação interprofissional, bastante discutida nos últimos anos e percebida cientificamente como um importante processo impulsionador do cenário atual das práticas em saúde, possibilitando aprendizagens compartilhadas e práticas colaborativas a partir do trabalho em equipe.

Desta forma, o modelo de formação a partir das Residências Multiprofissionais em Saúde, relativamente recente e em construção no Brasil, tem sido uma das grandes apostas de formação profissional em serviço. No entanto, os programas de residência multiprofissionais enfrentam numerosas problemáticas e as principais dificuldades encontradas são acerca da precariedade dos serviços onde os residentes são inseridos, a insuficiente ou verticalizada atuação pedagógica oferecida pelos programas e principalmente os desafios relacionados à atuação interprofissional, normalmente muito discutida nos estudos acerca das RMS e sobretudo vivenciada nos cenários de prática.

Contudo, observa-se neste estudo que, mesmo diante dos desafios postos aos profissionais residentes em seus espaços práticos e teóricos, estes ainda consideram que as RMS, na conjuntura do nordeste brasileiro, possibilitam o desenvolvimento de competências para uma prática colaborativa em saúde, onde os nutricionistas residentes apresentam compreensão similar aos dos outros residentes das demais categorias profissionais, permitindo a ampliação das discussões desta temática dentro do contexto da área da nutrição, particularmente pouco presente na literatura. 


\section{Colaboradoras}

de Albuquerque ERN, participou em todas as etapas do trabalho; de Santana MCCP participou da concepção e desenho do estudo, das revisões periódicas e da revisão da versão final; Rossit RAS participou da revisão da versão final do artigo.

Conflitos de interesses: As autoras declaram não haver conflito de interesses.

\section{Referências}

1. Brasil. Lei no 8080, de 19 de setembro de 1990. Lei orgânica da saúde. Dispõe sobre as condições para a promoção, proteção e recuperação da saúde, a organização e o funcionamento dos serviços correspondentes e dá outras providências. Diário Oficial da União. 20 set. 1990.

2. Aguilar-da-Silva RH. Educação interprofissional na graduação em saúde: aspectos avaliativos da implantação na Faculdade de Medicina de Marília (Famema). Educar em Revista. 2011; 39:159-175.

3. Silva JAM, Peduzzi M, Orchard C, Leonello VM. Educação Interprofissional e prática colaborativa na Atenção Primária à Saúde. Rev Esc Enferm USP. 2015; 49:16-24.

4. Araújo TAM, Vasconcelos ACCP, Pessoa TRRF, Forte FDS. Multiprofissionalidade e interprofissionalidade em uma residência hospitalar: o olhar de residentes e preceptores. Interface Comunicação, Saúde, Educação. 2017; 21(62):601-613.

5. Organização Mundial da Saúde. Marco para ação em educação interprofissional e prática colaborativa. Genebra: Organização Mundial da Saúde; 2010.

6. Peduzzi M, Norman IJ, Germani ACCG, Silva JAM, Souza GC. Educação interprofissional: formação de profissionais de saúde para o trabalho em equipe com foco nos usuários. Rev Esc Enferm USP. 2013; 47(4):977-983.

7. Batista NA. Educação interprofissional em saúde: concepções e praticas. Caderno FNEPAS. 2012; 2:25-28.

8. Câmara AMCS, Cyrino AP, Cyrino EG, Azevedo GD, Costa MV, Bellini MIB, et al. Educação interprofissional no Brasil: construindo redes formativas de educação e trabalho em saúde. Interface - Comunicação, Saúde, Educação. 2016; 20(56):9-12.

9. Brasil. Lei no 11.129 de 30 de junho de 2005. Institui o Programa Nacional de Inclusão de Jovens ProJovem; cria o Conselho Nacional da Juventude - CNJ e a Secretaria Nacional de Juventude; altera as Leis ns. 10.683, de 28 de maio de 2003, e 1 0.429, de 24 de abril de 2002; e dá outras providências. Diário Oficial da União. 01 jul. 2005.

10. Brasil. Ministério da Saúde. Residência multiprofissional em saúde: experiências, avanços e desafios. Brasília: Ministério da Saúde; 2006.

11. Casanova IA, Batista NA, Ruiz-Moreno L. Formação para o trabalho em equipe na residência multiprofissional em saúde. ABCS Health Sci. 2015; 40(3):229-233. 
12. Perego MG, Batista NA. Residência Multiprofissional em saúde: campo de aprendizagens compartilhadas com vistas à uma prática colaborativa. Anais do $12^{\circ}$ Congresso Internacional da Rede Unida. Revista Saúde em Redes. 2016; 2(1)Suplemento. Disponível em: http://conferencia2016. redeunida.org.br/ocs/index.php/congresso/2016/paper/view/2193

13. Brasil. Ministério da Saúde. Ministério da Educação. Residências Multiprofissionais em Saúde. [acesso em: 20 mar. 2017]. Disponível em http://portal.mec.gov.br/residencias-em-saude/residenciamultiprofissional

14. Brasil. Ministério da Saúde. Política Nacional de Alimentação e Nutrição. Brasília: Ministério da Saúde; 2013.

15. Brasil. Ministério da Educação. Conselho Nacional de Educação. Institui diretrizes curriculares nacionais do curso de graduação em nutrição. Resolução CNE/CES 5, de 7 de novembro de 2001. Diário Oficial da União. 9 nov. 2001; 1:39

16. Brasil. Ministério da Educação. Secretaria de Regulação e Supervisão da Educação Superior (Seres). Acesso em: 07 ago. 2017. Disponível em: http://portal.mec.gov.br/secretaria-de-regulacao-esupervisao-da-educacao-superior-seres/apresentacao

17. Vasconcelos IAL, Sousa MF, Santos LMP. Evolução do quantitativo de nutricionistas na Atenção Básica do Brasil: a contribuição dos Núcleos de Apoio à Saúde da Família e da Estratégia Saúde da Família de 2007 a 2013. Rev Nutr. Campinas. 2015; 28(4):431-450.

18. Rigon SA, Schmidt ST, Bógus CM. Desafios da nutrição no Sistema Único de Saúde para construção da interface entre a saúde e a segurança alimentar e nutricional. Cad Saúde Pública. 2016; 32(3). Disponível em: http://www.scielo.br/pdf/csp/v32n3/1678-4464-csp-32-03-e00164514.pdf

19. Brasil. Resolução CNRMS no 2, de 13 de abril de 2012. Comissão Nacional de Residência Multiprofissional em Saúde. Diário Oficial da União. 16 abr. 2012; 1:24-25.

20. Brasil. Conselho Nacional de Saúde. Resolução no 466 de 12 de dezembro de 2012. Diário Oficial da União. 13 jun. 2013; 1:59.

21. Oliveira CM, Batista NA, Batista SHSS, Uchôa-Figueiredo LR. A escrita de narrativas e o desenvolvimento de práticas colaborativas para o trabalho em equipe. Interface - Comunicação, Saúde, Educação. 2016; 20(59):1005-1014.

22. Rodrigues TF. Residências multiprofissionais em saúde: formação ou trabalho? Serv. Soc. Saúde. 2016; 15(21):71-82.

23. Costa MV, Patrício KP, Câmara AMCS, Azevedo GD, Batista SHSS. Pró-Saúde e PET-Saúde como espaços de educação interprofissional. 2015; 19(1):709-720.

24. Reeves S. Porque precisamos da educação interprofissional para um cuidado efetivo e seguro. Interface - Comunicação, Saúde, Educação. 2016; 20(56):185-196.

25. Recine E, Sugai A, Monteiro RA, Rizzolo A, Fagundes A. Saúde coletiva nos cursos de Nutrição: análise de projetos político-pedagógicos e planos de ensino. Rev Nutr. 2014; 27(6):747-760.

26. Fonseca AF, Corbo AMA. Organizadores. O território e o processo saúde-doença. Rio de Janeiro: EPSJV, Fiocruz; 2007. 
27. Silva CT, Terra MG, Kruse MHL, Camponogara S, Xavier MS. Residência multiprofissional como espaço intercessor para a educação permanente em saúde. Texto Contexto Enferm. 2016; 25(1). Disponível em: http://www.scielo.br/pdf/tce/v25n1/0104-0707-tce-25-01-2760014.pdf

28. Marra EMO, Faquim JPS, Carvalho JC, Nagao SM. Expectativa e satisfação dos usuários sobre os serviços oferecidos pelas equipes do Programa Saúde da Família. J Manag Prim Health Care. 2013; 4(3):158-168.

29. Brasil. Lei no 8.234 de 17 de setembro de 1991. Regulamenta a profissão de Nutricionista e determina outras providências. Diário Oficial da União. 18 set. 1991.

30. Souza SMB. A inserção da Nutrição nas Residências Multiprofissionais em Saúde [Trabalho de Conclusão de Curso de Especialização]. [Porto Alegre]: Universidade Federal do Rio Grande do Sul; 2013.

31. Santos IG, Batista NA, Devincenzi MU. Residência Multiprofissional em Saúde da Família: concepção de profissionais de saúde sobre a atuação do nutricionista. Interface - Comunicação, Saúde, Educação. 2015; 19(53):349-360.

Recebido: 07 de abril de 2018

Revisado: 04 de maio de 2018

Aceito: 22 de junho de 2018 
